\title{
Qu'est ce que la burnerie \\ selon Raphaëlle Giordano.
}

$$
\text { Dr. Amany M. Magdy }{ }^{(*)}
$$

\section{Abstract :}

La burnerie est une maladie sociale récemment parue dans les sociétés modernes due à une ébullition de comportements nuisibles. Notre recherche tenterait de répondre à la problématique suivante: Qu'est ce que la burnerie ? Quels sont les aspects de la burnerie ? Quels sont ses motifs, ses origines et Quel est le rôle de l'éducation dans le développement d'un caractère burné ? Comment prévenir la burnerie et comment en remédier ?

pour répondre à ces questions, notre recherche interrogera l'approche cognitive comportementale développé par le psychologue américain Jeffrey Joung. Cette approche comportementale étudie les aspects des différents schémas mentaux qui produisent les comportements nuisibles dans la société. Jeffrey Joung précise onze schémas de dysfonctionnements psychiques générant les différentes déviations comportementales dans nos sociétés modernes.

Nous allons essayer d'analyser les cinq comportements présentés par la romancière, tracer leurs aspects, chercher les raisons de leurs déviations. Nous allons tenter, par la suite, de les appliquer sur la théorie cognitive comportementale de Joung pour comprendre le rôle de l'éducation dans la propagation de ce genre de maladie sociale. savoir comment prévenir les attaques virtuelles de la burnerie sociale est l'objectif de la recherche.

${ }^{(*)}$ Département de Langue française - Faculté Al Alsun - Université de Minia 


\section{Avant propos :}

Raphaëlle Giordano est une écrivaine française contemporaine, spécialiste en créativité et développement personnel, artiste et peintre. Diplômée de l'école supérieure en arts appliqués, elle s'est également spécialisée dans la psychologie positive qui cherche à remédier tôt aux comportements nuisibles dans la société. Une femme douce, épanouie et surtout capable de diagnostiquer les maladies dont souffrent la société contemporaine.

Ses romans offrent à la littérature contemporaine une approche créative de développement personnel. En 2015, elle publie son premier roman: "Ta deuxième vie commence quand tu comprends que tu n'en as qu'une"1; un best seller mondial. A travers une histoire simple, elle guide son hérö̈ne stressée par un train de vie dur vers une méthode communicative calme et douce dans le but de retrouver son goût de la vie. Son second roman, objet de notre étude :" Le jour où les lions mangeront de la salade verte $^{\prime \prime 2}$ porte également un titre long avec un marqueur de temps clair qui aura plusieurs signification plus tard dans le roman. Quant à son troisième roman, nouvellement paru, intitulé : "Cupidon a des ailes en carton" ${ }^{3}$, la romancière s'y intéresse à apprendre au lecteur comment éviter les fautes régulières dans les relations amoureuses. Spécialiste en développement personnel, elle a jugé mieux vulgariser les méthodes et les bases cognitives et comportementales de développement personnel sous forme de roman pour diffuser ses idées plus largement et d'une manière moins rébarbative pour le lecteur. Giordano

1 Giordano (Raphaëlle), Ta deuxième vie commence quand tu comprends que tu n'en as qu'une, Paris, édition Eyrolles, 2015.

${ }^{2}$ ID, le jour où les lions mangeront de la salade verte, Paris, Eyrolles, 2017.

${ }^{3}$ Id, Cupidon a des ailes en carton, Paris, Eyrolles, 2019. 
développe dans ses trois romans un thème qui lui est cher à savoir comment retrouver le bonheur de vivre? Comment aider les gens à retrouver le chemin du bien-être et du bonheur?

\section{INTRODUCTION:}

Les sociétés développés s'intéressent actuellement à prévenir le stress qui règne dans les 4 coins du monde à cause du rythme de vie constamment en ébullition. Les travers sociaux se sont amplifiés à cause de la compétition à atteindre des scores plus élevés. Plus les exigences de la société exercent des pressions émotionnelles sur la personne, plus son comportement se transforme et se dégrade. Cette société dite moderne engendre par son rythme des comportements nouveaux.

La burnerie est un nom féminin dérivé du verbe anglais "burn", c'est à dire "incendier". L'incendie dans les relations humaines ne peut que traduire un épuisement émotionnel dû à l'incompréhension. L'irritabilité dans les relations sociales engendre un genre de stress qui devient chronique. Et lorsqu'on parle d'incendie dans les comportements sociaux, cela mène à retenir l'attention.

Le petit Robert et le Larousse illustré ne portent aucune signification du mot, ce qui traduit sa parution récente dans la langue française.

Qu'est ce que la burnerie ? Quel genre de maladie sociale est-elle ? Quels sont ces aspects ? Comment reconnaître une personne qui développe un comportement burné dans la société ? Comment prévenir de tomber proie à la burnerie conjugale ou professionnelle ? Comment s'immuniser contre ce genre de travers sociaux ? Quelles solutions nous présente la littérature pour nous éclaircir sur cette maladie sociale ? Telles sont les raisons qui ont suscité notre attention pour chercher à comprendre les raisons de la 
propagation de la burnerie dans les sociétés modernes. L'agressivité, l'attaque inappropriée, infondée, injuste, et la tendance au jugement négatif semblent être quelques caractéristiques de la burnerie dont souffre la société contemporaine à des degrés différents. Le manque de temps, de tolérance, et d'éducation aussi ont probablement engendré ce genre de comportements agressifs des uns envers les autres.

Giordano s'intéresse, dans ses romans, à étudier les travers de la société. Loin de l'analyse psychologique proprement dite, elle inaugure un champ de traitement comportemental tracé sous la lignée de la psychologie positive; celle intéressée par la prévention des maladies. La psychologie positive cherche à faire le diagnostic des travers de la société, essayer d'y remédier avant d'atteindre les phases de psychose ou de névrose. Nous nous sommes intéressée à étudier cette nouvelle approche vue son utilité et l'impact positif qu'il peut jouer dans l'amélioration des comportements des gens dans la société. Cette approche vise fondamentalement à éveiller les consciences, à secouer les habitudes néfastes et ancrées, à sensibiliser la personne au mal qu'il cause intentionnellement envers autrui.

Nous allons essayer d'aller un peu plus profondément pour comprendre la source qui a engendré la burnerie chez chaque caractère présenté par la romancière. Nous allons par la suite présenter quelques éléments non exhaustifs de sa thérapie comportementale pour montrer le fonctionnement de l'approche. Nous nous sommes basées sur l'approche cognitive comportementale du psychologue américain Jeffrey Joung. Le psychologue et son groupe de travail ont recensé onze schémas d'inadaptation comportementale. Nous allons découvrir comment le thème de la burnerie repose sur un schéma intitulé : " Tout nous 
est dû". Nous allons voir comment les personnes qui développent ce schéma blessent insensiblement leurs entourages, comment ils s'arrogent tous les droits comme s'ils étaient uniques au monde.

Giordano présente cinq personnages burnés, chacun d'eux est coincé dans une dédale de problèmes qu'il a lui même tissé, par incompréhension, entre lui et son entourage. L'approche de Joung explique parfaitement les cinq cas de burnerie présentés par la romancière. Le dernier point que nous allons traiter serait de chercher les raisons profondes cachées sous l'écorce de la dureté et les moyens d'y remédier. Nous allons découvrir le but final de l'approche et les moyens de rétablissement du moi sain empoisonné par une éducation inadéquate.

Notre roman n'est pas un roman de psychologie,aucune analyse de maladie psychique. Il n'est pas non plus un roman didactique. C'est un roman simple dans sa structure narrative et son intrigue. La forme du roman est celle de la fiction avec des personnages, des marqueurs temporels, et des descriptions. Une trame narrative simple, une nouvelle forme inédite : mi roman, mi guide de développement personnel. Le roman présente une forme de pensée argumentative implicite; une certaine forme d'apologue ${ }^{4}$ qui critique la société dans le but de la réformer.

Romane Gardner, alter ego de la romancière, jeune femme d'une trentaine d'années, dirige une association nommée "SUP DE BURN" où elle reçoit des personnes en difficulté

${ }^{4}$ L'apologue est un récit narratif qui sert à illustrer une morale. II a un but argumentatif et didactique comme les Fables de la Fontaine au 17ème siècle ; comme le conte philosophique " Candide"de Volaire au 18eme siècle. Les récits utopiques qui ont pour but de critiquer les travers de la société et donner une leçon de morale font également partie de L'apologue. 
morale, sociale ou psychique. Payée par des entreprises, elle aide les salariés à développer leurs inventivités en groupe et résoudre leurs problèmes psychiques. Elle entreprend d'analyser avec eux en douceur les raisons de leurs blocages respectifs. Elle leur présente un programme de "Relooking intégral" dans le but de dynamiser leurs énergies et de développer leurs inventivités. Sa méthode de travail repose à faire reconnaître à ses collaborateurs où réside le mal. Quel comportement nuisible sont-ils en train de répéter sans cesse dans leurs vies quotidiennes et qui a abouti à ce blocage relationnel. Cette méthode dite cognitive repose sur des exercices accompagnés; chaque exercice remplit un objectif précis. Romane Gardner applique les étapes curatives entreprises par le psychologue GEFFREY YOUNG. Spécialiste en science du comportement, Young trace les étapes d'une cure comportementale, caractérielle et existentielle. Young a mené ses expériences dans les laboratoires. Raphaëlle Giordano a décidé de diffuser ces méthodes curatives au grand public. La forme romanesque semble être plus divertissante.

Giordano part du postulat que L'HOMME dans la société est un lion pour L'HOMME :"l'Homme est un lion pour l'homme. Et les lions ne s'embârrassent pas de délicatesse. Sûrs de leur bon droit, ils imposent leurs vues sans conscience de leur Égocentrisme et de leur appétit excessif pour les rapports de force". (Giordano, le jour où les lions mangeront de la salade verte, Eyrolles, Paris, 2017,p.1)

C'est avec cette métaphore ironique que Raphaëlle Giordano a commencé son roman. Un clin d'œil ironique lancé au lecteur pour l'inviter à prendre conscience de ses mauvais caractères, les dénoncer, s'en moquer avant d'entreprendre la tentative du changement comportemental.

Spécialiste en créativité, Giordano prend soin de susciter l'intérêt de son lecteur à travers l'invention des 
néologismes; ce qui l'invite à méditer . Dans son premier roman, elle a inventé le métier de "Routinologue" qui accompagne le personnage principal dans sa tentative de rendre sa vie moins routinière afin d'atteindre le bonheur . Dans son troisième roman, elle a inventé le nom "Amourability" pour désigner la capacité d'aimer.

Dans le second roman, elle utilise le terme de "burnerie" pour désigner les comportements agressifs et les comportements nuisibles considérés comme harcèlement moral et psychique en société. Selon Giordano, La burnerie est un nom féminin qui désigne l'ensemble des comportements nuisibles qui portent atteinte à la sensibilité des autres. Si Marie-France Hirigoyen dans son ouvrage " le harcèlement moral et la violence perverse au quotidien" 5 a expliqué ce qu'est le harcèlement et les méthodes de le braver, Raphaëlle Giordano décrit ici les personnes qui produisent ce genre de harcèlement sans se soucier des sentiments d'autrui.

La burnerie se dévoile dans les "attentats à la sensibilité comme le manque de tact, le manque d'écoute, et le manque d'empathie. La burnerie est un penchant pour l'agressivité qui atteint "l'autre" sans se soucier de ses sentiments, un penchant à blesser sans s'en rendre compte, à critiquer sans fondement, injustement."(corpus p. 9).

Le harcèlement moral se trouve sous plusieurs formes: le harcèlement conjugal, professionnel scolaire et parental.

${ }^{5}$ Cf. Hirigoyen (Marie-France), le harcèlement moral et la violence perverse au quotidien, Paris, Étude (poche), 2018. Hirigoyen a expliqué la différence entre le pervers narcissique et le manipulateur en analysant des cas pathologiques de sa clinique. Elle a présenté les caractéristiques du pervers narcissique et les méthodes de braver son hégémonie sur l'entourage. Le manipulateur et le pervers tirent une joie malicieuse en manipulant leur entourage, cependant la personne burnée n'est pas conscient du malheur qu'il propage autour de lui. Le premier blesse avec préméditation consciente, le second en est inconscient. 
Giordano nous peint un tableau où figurent trois genres de harcèlement.

\section{ASPECTUALISATION DES COMPORTEMENTS}

\section{BURNES :}

1) Égocentrisme ou inflation de l'Ego :

l'incipit du roman présente une scène de tauromachie où le duel entre un taureau et son toréador résume un duel des ego. Giordano présente deux instincts "mâles" qui s'efforce chacun de vaincre l'autre après l'avoir provoqué; une schématisation de ce qui ce passe dans les relations sociales. Chacun s'applique à vaincre l'autre et prouver qu'il a toujours raison.

"Sa puissance mâle incarnant, malgré elle, le Mal en puissance... (la puissance du taureau) (....) Duel des ego. Orgueil mâle piqué au vif par les banderilles. Naseaux et narines frémissent d'un même désir de vaincre. Le toréador anime alors d'un geste leste le drapeau rouge, comme un fulgurant trait de pinceau provocateur". (corpus p. 7)

Selon Giordano, la burnerie est un caractère purement masculin. Cet apanage de l'agressivité masculine est due au flot de l'hormone "testostérone" produit en profusion par le cerveau masculin. La romancière raisonne ainsi:

"les comportements burnés sont bourrés de testostérone! Et parce que la burnerie est un concept très masculin en soi". (corpus. P.23)

Dr. Louann Brizendine, dans son ouvrage intitulé "les secrets du cerveau féminin" avait d'ailleurs prouvé ce raisonnement :

" Il existe toutefois une énorme différence dans la quantité de testostérone disponible pour" exciter" le cerveau : les hommes en possèdent de dix à cent fois plus que les femmes". (Brizendine, les secrets du cerveau féminin,Paris , édition Bernard Grasset, p. 159)

Cette même étude prouve que " Hommes et femmes produisent de la testostérone, mais chez l'homme la production est dix fois plus importantes(...)" (Brizendine, p. 104) 
Michael Kaufman a également étayé l'agressivité masculine dans son article : "Les 7 P. de la violence masculine""6 . Le cinquième $P$ qu'il a intitulé "armature psychique (mentale) de la virilité" montre que "ce processus complexe et particulier de développement psychologique (manière d'éduquer les garçons) entraîne une capacité d'empathie (ressentir ce que les autres éprouvent) amoindrie et une incapacité à percevoir les besoins et les sentiments des autres (...). " ( Kaufman, Toronto, Canada, 1999, P. 4)

L'écrivaine rend l'éducation responsable de ce goût inexplicable pour l'agressivité chez le mâle. S'adressant essentiellement à un public masculin, Romane Gardner, l'alter ego de l'écrivaine explique :

"Des siècles d'héritage culturel et d'éducation burnée à votre actif! Vous avez été élevés au biberon du pouvoir, de la domination, de la force, du machisme, c'est difficile pour vous d'enrayer d'un claquement de doigts des comportements aussi enracinés". (corpus. P.23)

Jeffrey JOUNG,dans son ouvrage, "je réinvente ma vie" a décrit ce genre de comportement agressif mais surtout égoïste:

"Vous êtes égocentrique, nul ne vous intéresse que vous même." (Joung, Je réinvente ma vie, éditions de l'homme, Canada, 2013,p.337 ) Il ajoute: "Les besoins d'autrui ne vous intéressent pas. Vos besoins passent avant les leurs. Vous les blessez". (Joung, je réinvente ma vie, p. 335)

C'est le cas des cinq personnages présentés par Giordano dans son roman. Tous souffrent d'un égö̈sme enflé et d'un manque d'empathie envers les autres.

${ }^{6}$ Cf. Kaufman (Micheal), Les sept "P" de la violence masculine, Toronto, Canada, 1999. P. P. 1-8 . Kaufman à précisé 7 raisons qui rendent les hommes plus violents et agressifs que les femmes: 1)Le pouvoir patriarcal. 2) Le sentiment du Privilège dû. 3) La permission. 4) Le paradoxe du pouvoir des hommes. 5) l'armature psychique (mentale) de la virilité. 6)La masculinité comme cocotte minute psychique. 7) Les expériences du passé. 
2) Rigidité émotionnelle et sentimentale :

Le caractère burné est un caractère trop centré sur sa propre personne, incapable d'écoute, impatient, préfère promulguer des jugements critiques et acerbes sans fondements.

Maximilien ${ }^{7}$ Vogue, un P. D. G. d'une prestigieuse compagnie de cosmétique est un typique de la burnerie de pouvoir. Rigide, autoritaire, impassible, Maximilien est dur envers les autres. Sa rigidité émotionnelle l'assimile à un lion: "Maximilien se leva(...) tel un lion en cage". (corpus p. 26),

Face à l'appel de sa sœur jumelle le suppliant de lui venir en aide, Maximilien lui prête une oreille sourde:

"Il leva vers elle son visage des mauvais jours, celui où la ride du lion durcissait son expression et où son regard froid pouvait vous pétrifier." (corpus. P. 18)

Cette incapacité à sentir la douleur même de ses proches a guidé sa sour au suicide. Giordano présente Maximilien comme l'archétype de la burnerie de pouvoir :

" un instinct de domination et un sentiment de supériorité plus ou moins exacerbés, ainsi qu'un penchant naturel pour les jeux de pouvoir ou les rapports de force". (corpus p. 9)

Maximilien a dû adhérer forcément au programme de changement ou "relooking comportemental" après avoir subi le choc du suicide de sa sour.

Le cas de Maximilien est exactement décrit par Joung," votre conjoint, votre famille, vos amis ou vos enfants vous quittent, vous en veulent ou rompent tout contact avec vous parce que vous les maltraitez, que vous êtes injuste ou égoüste."(Joung, je réinvente ma vie, p. 335.

Et c'est effectivement ce qui s'est passé avec lui lorsque sa sour lui a lancé maints appels au secours avant de tenter le suicide. Elle a refusé, même, de le revoir après son

1) Nous notons que le choix du nom est judicieux parce que ce Président directeur général est un caractère ultra burné. 
rétablissement. La secrétaire de Maximilien, trop dévouée depuis des années , a dû elle aussi le quitter :" vos exigences épuisent vos proches". (Joung, Je réinvente ma vie,édition de l'homme, Canada, 2013,p. 335)

Celle-ci a refusé de devenir une "cat-sitter" pour le chat offert par Romane. Elle a jugé que son directeur abuse de sa gentillesse. Comme si Maximilien était présent en face du psychologue Young qui lui explique:

" les gens de qui vous dépendez finissent par se lasser ou s'irriter de votre dépendance et de vos caprices, ils vous quittent, vous congédie ou refuse de vous venir en aide." (Young, je réinvente ma vie, p. 335).

3) Intransigeance / intolérance :

La troisième caractéristique de la burnerie est l'intolérance envers les autres: une rigidité du comportement et une incapacité à se mettre à la place de l'autre pour tolérer ses défauts. La romancière met en place un caractère rigoureux, impassible mais surtout intolérant aux sujets des femmes.

Bruno, ou monsieur Robot, manager dans une grosse entreprise de consommation dirige une équipe de huit femmes qui l'ont dénoncé pour une "Rigidité" de comportement. Elles ont dénoncé ses harcèlements quotidiens et son manque de souplesse à leurs égards. La critique négative, le jugement injustifié, et une attitude crispée ont poussé ses employées à le dénoncer à la direction de l'entreprise. En voilà un exemple de ses critiques:

"Les femmes au travail, au premier coup de chauffe, elles perdent leurs moyens, (....) puis après, elles vous font leur crise de calgon, se mettent à pleurer". (corpus p. 75)

Joung décrit ce genre de comportement en expliquant :" Vous abusez de votre entourage, vous l'humiliez ou vous le dénigrez. (...) Vous n'éprouvez aucune empathie pour les gens qui vous entourent. Ils croient que vous ne les comprenez pas et que vous ne vous souciez pas de ce qu'ils ressentent." (Joung, je réinvente ma vie, p. 335). 
C'est pour cette raison que ses employées l'ont dénoncé à la direction. Celui-ci ci a dû suivre le programme de déburnerie avec Romane Gardner, parce que ses dirigeants l'ont forcé à s'y inscrire. Ce fût un second exemple de burnerie de pouvoir ou de harcèlement moral au quotidien .

\section{4) Critique et dévalorisation de l'Autre :}

Nous pensons que la personne égoïste, trop plein de lui même se trouve également incapable d'apprécier les qualités des autres. Patrick : un caractère de burnerie conjugale. Quoiqu'il aime sa femme, il ne cesse, jour et nuit de la harceler moralement en dévalorisant ses tentatives de maintenir l'équilibre entre l'entretien de sa maison et son travail à domicile. Elle le quitte après vingt cinq ans de mariage. Giordano dresse le tableau de cet harcèlement conjugal:

"(...) elle avait été blessée par le manque d'intérêt total qu'il portait à l'activité qu'elle essayait de développer, (...) et cette manière vexante qu'il avait de lui faire sentir qu'elle ne gagnait pas assez d'argent." (corpus p. 138)

Ce sont exactement ce genre de harcèlement, de critiques blessantes que la romancière veut souligner pour tirer au clair leurs conséquences négatives sur la société :

" (ce sont de) "Petits attentats à la sensibilité" trop souvent perpétrés (manque de tact, manque d'écoute, mesquineries diverses) la regrettable propension à l'agressivité facile ou gratuite, sans oublier la mauvaise foi en toute bonne foi, si tristement répandue. " (corpus p. 9).

Alors, Sous le choc, Patrick consulte pour comprendre quelle faille comportementale peut-elle lui reprocher. Il n'arrive pas à comprendre, à ce stade que :

" votre conjoint, votre famille, vos amis ou vos enfants vous quittent, vous en veulent ou rompent tout contact avec vous parce que vous les maltraitez, que vous êtes injustes ou égoüste." (Joung, je réinvente ma vie, p. 335) 
5) Hégémonie et amour de la possession:

Malgré la tendance masculine vers le harcèlement de l'entourage, les femmes n'en sont pas exclues. Deux figures ont succombé, elles aussi à la burnerie.

Émilie, dame bourgeoise possessive, vient consulter pour découvrir sa part de responsabilité dans la fugue de son fils:

"Je viens ici, parce que .... mon fils a fait une fugue récemment... Et je veux comprendre ma part de responsabilité". (corpus p. 39)

De son point de vue de bourgeoise possessive, elle veut contraindre son fils à suivre des études prestigieuses correspondant à leur caste riche:

" Je voyais pour lui un avenir si brillant! J'ai voulu l'obliger à suivre la même voie que moi, à suivre des études prestigieuses, et je n'ai pas su voir sa nature profonde". (corpus p. 65)

Son fils, jeune homme débordant de vivacité et déterminé à tracer seul la trame de sa vie refuse de se plier aux exigences de sa mère et quitte la maison:

"Non, je ne reviendrai pas. Je ne peux plus supporter que tu essayes de diriger ma vie, que tu m'imposes des choix qui ne sont pas les miens. On n'est pas fabriqué pareil. Pourquoi veux tu m'obliger à te ressembler? Quand est ce que tu vas enfin arrêter de vouloir tout contrôler et surtout me contrôler, moi ?" (corpus p. 66).

Nathalie, jeune femme d'une trentaine d'années, licenciée de son travail malgré une énergie débordante et une hyperactivité voyante, vient consulter pour comprendre en quoi son comportement peut-il gêner ses collègues. Surprenante, effrayante, elle commence toutes ses phrases par :"Moi, je...." ce qui traduit un vouloir excessif de se montrer, de prouver sa présence. Mais à force de toujours vouloir être remarquable, elle finit par rebuter son entourage :

"La jeune femme n'avait pas conscience de sa burnerie moi-je-iste et de sa panoplie de comportements exaspérants pour les autres: tendance à parler trop haut, trop fort, à monopoliser l'attention, à faire son 
intéressante, à en mettre plein la vue... Une personnalité cent mille volts! Attentiopathe et énergiphage" (corpus p. 58)

Young décrit ainsi le cas de Nathalie, comme s'il s'adresse à elle en personne: " Vous avez depuis toujours de la difficulté à gérer vos comportements et vos émotions. Vous parvenez difficilement à dominer votre impulsivité. Vous laissez vos désirs et vos émotions dicter vos actes sans égard aux conséquences." ( Joung, Je réinvente ma vie, p. 328)

Comme s'il expliquait, d'une manière directe et franche au personnage du roman, les problèmes reliés à sa discipline personnelle:

" Le sentiment que tout vous est dû vous porte à les (vos besoins) exprimer à l'excès. Vous ne savez pas vous retenir, (...). Votre vie ne devient intolérable que lorsque vous ne parvenez plus à éviter les conséquences fâcheuses de votre comportement, par exemple, quand on vous congédie parce que vous faites mal votre travail ou quand votre conjoint menace de vous quitter". (Joung, je réinvente ma vie, p. 330).

Ce cas est typiquement celui de Nathalie et de Patrick. Nathalie a été effectivement surprise d'être licenciée, Patrick a été déstabilisé par la fuite de sa femme.

C'est ainsi que Raphaëlle Giordano dresse le tableau des comportements burnés qu'elle essayera de guider vers plus de compréhension de leurs propres lacunes respectives. Alors, récapitulons les cas de harcèlement :

"un patron sans arrêt sur votre dos (Bruno) pour vous mettre la pression, un conjoint qui a le dénigrement facile (Patrick) (...) une bonne copine qui, en société, accapare toujours l'attention et avec qui vous ne pouvez pas en placer une (Nathalie), un parent qui juge systématiquement vos décisions ou votre manière de faire (Émilie). " (corpus p. 22)

Il paraît donc clair que chaque caractère a dû forcément aller consulter une experte en développement personnel lorsqu'il a été bloqué dans ses relations les plus étroites soit sur le plan conjugal, soit sur le plan professionnel.

Après avoir passé en revue les cinq problèmes, Romane met en place une méthode de prise de conscience. Elle guidera 
ses patients, non sans effort, à comprendre pour quelles raisons sont-ils arrivés jusque-là.

Nous notons que le but de la transmission de cette approche de développement du comportement dans un roman est de critiquer les points faibles de la société. La romancière vise à rendre les personnes plus compréhensives de leurs propres conduites. Elle tente de rendre plus fluide la communication entre les membres de la société,de rendre leurs trains de vie moins stressants. La fonction de la littérature rejoint ici celle du psychanalyste qui dissèque les maladies psychiques régnant dans la société dans le but de les analyser avant de tenter $y$ remédier.

La théorie du comportement repose sur des techniques motivationnelles dans le but d'améliorer la qualité du style de vie. Young explique :

"Dans notre approche centrée sur les schémas, nous recourons à des techniques cognitives, behaviorales, existentielles-humanistes, psychanalytiques, interpersonnelles et au travail sur l'enfant intérieur". (Joung, je réinvente ma vie. $P .353$ )

Nous déduisons que si Jeffrey Joung a mené ses expériences entre les murs des laboratoires, Raphaëlle Giordano en a transmis l'application à travers le roman. Les techniques de prise de conscience : méthodes cognitives:

La réactivation des cinq sens.

1) L'ouïe : apprendre à se décentrer.

Pour faire sauter leurs verrous intérieurs et casser l'écorce de leurs carapaces trop dur, Romane lance un sujet de discussion et invite ses collaborateurs à exprimer ce qu'ils en pensent. Elle enregistre leurs conversations, puis elle la leur fait entendre.

À la surprise de tous, ils découvrent pour la première fois comment ils réagissent agressivement, chacun, à la pensée de l'autre. Leurs expressions faciales, intonatives et 
corporelles trahissent un fond de pensée agressif. Un ton acerbe, des jugements et des critiques infondées dénotent un manque d'écoute et une tendance facile à se croire supérieur. L'arrogance de leurs comportements traduit leurs impatiences à écouter le point de vue de l'autre, ce qui les rend incapable de tolérance et d'empathie.

\section{2) La vision:}

Romane accompagne ses stagiaires à assister à une projection sur les mystères de l'univers à la cité des sciences. Indirectement, elle voulait les inviter à voir clair le vrai volume qu'ils occupent dans l'univers. Lorsque la galaxie compte entre cent et deux cent milliards d'étoiles et que l'univers peut compter quelques cent milliards de galaxies, alors les gens à ego enflé peuvent repenser leurs places dans cet univers:

"Quand on a développé certains travers burnés, on a tendance à être autocentré, à se croire le centre du monde. Le film que vous avez vu au planétarium vous invite à garder en mémoire notre petitesse : nous sommes d'infimes petits points qui s'agitent dans $l$ ' infiniment grand (.....) cela aide à rester humble, n'est ce pas ?" ((corpus p.76)

Une question lancée à la fin de la séance joue le rôle d'un éléctro- choc. Chacun est donc invité à reconsidérer sa place dans cet univers infiniment grand. Se décentrer, c'est à dire réaliser qu'il y a un Autre qui peut, lui aussi, avoir un point de vue différent:

"Le monde, votre monde n'est pas le même que celui de votre voisin. Vous devez prendre conscience que chacun perçoit le monde de son point de vue. Or, croire que son point de vue est le meilleur conduit à bien des problèmes de communication et d'entente avec les autres. " ( corpus p. 77)

Pour atteindre un stade d'harmonie avec le monde environnant, ils doivent réaliser que cet Autre existe, il faut lui reconnaître le droit d'avoir un point de vue différent :

" pour élargir votre vision du monde, il vous faut changer de point de vue, vous décentrer, changer de place et de perspective..." (corpus p. 77) 
Elle leur propose par la suite, de dessiner pour visualiser. Chacun exprime sa position par rapport au monde par le dessin: une activité leur permettant de comprendre le déséquilibre relationnel dû à leur mauvais caractère :

" l'objectif de cette activité, c'est de faire apparaître les" dysfonctionnements, incohérences, déséquilibres relationnels d'un plan de vie". (corpus p. 78)

A partir de là, les personnages de Giordano commencent à réaliser le dysfonctionnement de leurs comportements envers leurs entourages.

La psychologie positive repose sur l'idée que chaque comportement peut être compris par une prise de conscience du sujet. La stratégie cognitive utilisée par Giordano vise à atteindre cette compréhension de soi dans l'objectif de faire changer ces comportements nuisibles pour améliorer leur style de vie.

Le "troc fauteuil" est une activité de travestissement de rôle. Romane Gardner invite ses collaborateurs à passer une journée dans la peau d'une personne de leur entourage pour assumer le mêmes coups qu'ils cèdent inconsciemment à leurs proches. Cette activité vise à les sensibiliser à la différence entre le management lucide basé sur la compréhension mutuelle et "le management autocraticotyrannique". (corpus $p$. 111). Pour mieux rendre compte de cette expérience, elle leur propose de filmer leurs journées respectives au moyen d'une caméra miniature avec un micro intégré.

3) le toucher ou la réactivation du sensoriel:

Pour cultiver le décentrement d'un "moi" trop égoïste, la romancière conseille de " mettre sur "off" ses pensées et sur "on" ses cinq sens, l'un des secrets pour se connecter au quotidien, à la joie de vivre et à la sérénité. " (corpus p. 164)

Pour pouvoir se décentrer,oublier un peu son" moi" et aller vers les autres d'une manière authentique, la relaxation 
sensorielle est d'une utilité infaillible. Il fallait tout d'abord être bien dans sa peau. Pour Maximilien, le PDG enfoui dans la carapace de la dureté, elle lui conseille une expérience unique d'isolation sensorielle. Flotter dans de l'eau iodée pendant une heure l'a aidé à relâcher autant ses muscles que ses nerfs. En se laissant bercer par le mouvement doux de l'eau Maximilien fût surpris que " c'est un moment de relaxation unique dont les effets bénéfiques ne sont plus à prouver sur la gestion du stress, la qualité du sommeil, les capacités de concentration ou la créativité". (corpus p. 159.)

Miraculeusement, cette expérience a réussi à le détendre nerveusement et émotionnellement:

"probablement, un instant, Max. Songea que ce qu'il ressentait devait être proche de l'état de quiétude du milieu intra-utérin. Oui, il était amniotisé !". (corpus. P. 161)

Patrick et Bruno ont fait des séances de massage pour relâcher les muscles et les nerfs:

" Un incroyable massage à quatre mains (...) afin qu'il reprenne contact avec son corps en surpoids qu'il avait tendance à négliger". (corpus p. 164)

Quant à Nathalie qui adore accaparer l'attention de son entourage, "elle avait participé à une séance collective de chants(...), afin de sentir par le chant le bénéfice invisible à créer du lien et à être à l'écoute des autres, en harmonie." (corpus p. 164).

À mi-chemin de son programme de Relooking comportemental, Romane aide chaque collaborateur à découvrir où réside son mal-être. Elle fait découvrir à Maximilien que " sa burnerie de pouvoir, évidente, avait mis un écran entre lui et ses émotions, de telle sorte qu'il ne recevait plus certains signaux d'alerte. Il avait si bien construit sa carapace qu'il était certes mieux protégé, mais aussi coupé des autres". (corpus p. 133)

Romane explique également à Émilie les fautes commises dans l'éducation de son fils:

"Vous projetiez sur votre fils votre vision du monde et vos désirs, en oubliant de vous mettre à sa place. (...) Il faut décentrer votre univers et 
accueillir les points de vue différents du vôtre. Vivre et laisser vivre..." ( corpus p. 106)

Elle l'invite, par la suite, à prendre soin d'elle même dans une tentative symbolique de réorienter son énergie loin de son fils et l'inviter à se couver elle-même. Elle lui explique comment mettre au point une sorte d'égoïsme éclairé, prendre soin de soi, plutôt que de s'impliquer dans la vie de son fils.

Romane montre à Nathalie que sa façon de toujours vouloir accaparer l'attention finissait par être étouffante. Elle ne laisse pas la place aux autres pour exister en sa présence :

"Nathalie, si vous réalisez peu à peu que, dans votre cas, il est important de laisser plus de place à l'autre dans la relation, si vous arrivez à ressentir les bénéfices d'être mieux à l'écoute et dans l'échange, si vous comprenez que se mettre en valeur ne veut pas forcément dire se mettre en avant, alors l'attitude de vos interlocuteurs va changer elle aussi. " (corpus p. 58)

Le fait de ne pas se soucier ni des sentiments ni des paroles de son entourage, a provoqué une répulsion à son égard. Elle a perdu sa fonction et ses amis parce qu'elle ne se soucie jamais de leurs sentiments. Elle veut toujours se montrer, être vue, entendue. La raison probable de cette déviation comportementale revient à son statut social de célibataire. Sans mari, sans enfants, sans relation familiale, elle a développé petit à petit un comportement égoïste qui ne cherche qu'à se montrer. Par conséquent, elle a perdu ses amis et a gagné la répulsion de ses collègues et directeurs.

Romane fait remarquer à Bruno que son intolérance aux sujets des ses collaboratrices était dûe à un manque de souplesse et de flexibilité :

"Lui, rigoureux,organisé, méthodique et efficace pouvait certainement devenir tranchant, tyrannique d'exigence, castrateur, colérique...". (corpus p. 91) 
Il paraît clair que le manque de flexibilité était dû à sa condition sociale d'homme célibataire,lui aussi, il consacrait tout son temps et toute son énergie à sa carrière. Il n'avait pas songé que cette disponibilité excessive n'était pas le cas de ses collaboratrices. Il devait prendre conscience "des différences structurelles de personnalité d'un individu à l'autre". (corpus p. 91)

Quant à Patrick, il était le premier à reconnaître seul jusqu'à quel point il a été dur avec sa femme malgré son amour. L'expérience du "Troc fauteuil" lui fait découvrir visuellement son injustice envers elle:

"J'ai dû souvent être injuste avec elle. Je ne réalisais pas la lourdeur de sa tâche. Je pensais que c'était facile de rester à la maison et de " bossouiller" avec sa petite entreprise. Je la taquinais souvent avec ça." (corpus p. 104)

Jusqu'à lors, Romane a travaillé sur la cognition des sentiments inextricables chez ses stagiaires. Elle a travaillé à secouer leurs consciences à travers cette méthode cognitive. Voir clair et se responsabiliser vis à vis de ses actes aident probablement à un changement comportemental prévu.

Techniques de changement du comportement:

Apprentissage de l'altérité :

Ces personnages burnés étaient emprisonnés dans un carcan de stress, une ébullition sociale, et une dureté émotionnelle. La thérapie cognitive comportementale les guide à développer leur sensibilité rigide . Romane va les aider à débloquer leurs émotions emprisonnées sous la carapace de leurs schémas pathologiques:

"Cultiver la sensibilité, le tact, la gentillesse et l'harmonie". ( corpus p. 187)

Elle essayera de "faire grandir son acuité émotionnelle, cela revient à accéder à un septième sens. Cela vous donne une dimension humaine encore plus importante. C'est comme passer d'un monde en noir et blanc à un monde en couleurs! Acquérir une nouvelle dimension 
émotionnelle, c'est devenir plus fort, plus vivant et encore une fois, plus humain". ( corpus p. 187)

Romane les pousse à organiser une journée de bienfaisance, chacun serait responsable tout d'abord de prendre soin d'un petit animal, pour être capable de retrouver la joie enfantine. L'apprentissage de l'altérité passe d'abord par la capacité, mais surtout, le vouloir de donner de sa propre personne, de son temps pour canaliser la sensibilité nouvellement requise vers la tendresse. Romane offre à Maximilien un petit chat sans défense. II serait responsable d'une créature fragile dont la vie ne dépendra que de ses soins:

"Cette petite boule de poils réussirait-elle à attendrir son coeur de dur à cuir ? Toucher sa corde sensible, libérer sa part de tendresse, (...)." (corpus p. 170)

Romane espère "toucher une corde de sa sensibilité restée jusqu'à lors atone". ( corpus p. 191)

Quant à Bruno, il reçoit un petit lapin sans défense pour l'initier à partager la tendresse. Sans le savoir "elle (Romane) venait d'exaucer un rêve de gosse." (corpus p. 167)

Patrick reçoit un couple de petits oiseaux pour lui offrir tous les jours la vision d'un amour tendre et inconditionnel pour évoluer sa conception de la vie de couple.

Prendre soin de ces créatures fragiles leur permettrait de redevenir enfant: "Libre de transgresser les codes rigides de l'adulte, de scier les barreaux du politiquement correct et d'oser jouer." (corpus p. 244)

Après les soins prodigués forcément à ces créatures, l'étape suivante fut de les inviter à semer la joie dans la vie d'enfants orphelins. Chacun doit consacrer une journée entière dans la compagnie des orphelins. Après le contact direct avec ceux-ci, Maximilien réalise avec stupeur que Donner est un bonheur en soi: 
"Dire qu'il avait intégré ce programme dans l'espoir de se changer, lui. Il réalisait contre toute attente, qu'en se changeant lui, il pouvait aussi changer la vie d'autres personnes." ( corpus p. 257)

\section{Apprentissage de la spontanéité :}

Romane leur lance ce défi : elle leur retire leurs portables pendant une journée complète. Avec beaucoup de résistance, le groupe accepte, mais à contre cour de se libérer de leurs smartphones. Romane leur explique qu'il faut apprendre à se connecter aux humains pour goûter le vrai calme, qui permet par la suite de se reconnecter à soi: "Elle expliqua brièvement comment ces objets censés n'avoir qu'une vocation fonctionnelle étaient devenus pires que des objets transitionnels: des objets tyrans imposants leur dictature d'omniconnexion, vampirisant les temps de présence à l'autre, créant une dépendance néfaste, une addiction renforçant l'anxiété (....), maintenu malgré lui dans un constant état de vigilance". (corpus p. 224).

Laisser de côté son portable initie la personne à être moins connecté au monde virtuel, loin sensiblement de ses proches. Le virtuel tue la spontanéité d'un geste ou d'un sentiment qu'on peut prodiguer par amour à l'entourage. Cultiver cette spontanéité réduit les risques de vouloir tout contrôler et tout juger. Romane invite ses collaborateurs à se libérer du joug du jugement, de l'œil critique posé constamment sur autrui:

"Affranchissez-vous du jugement, de vouloir tout contrôler, du regard des autres, ( soyez) prêt à oser s'amuser, à prendre du plaisir." (corpus p. 224)

Jusqu'à présent, Romane a joué le rôle d'un thérapeute actif qui guide le groupe à reconnaître chacun sa part de responsabilité dans chaque blocage relationnel. A travers ces exercices de restructuration comportementale, les personnages du roman devraient modifier et développer leur habileté communicative.

Les schémas pathologiques: 
Dans son ouvrage intitulé "Thérapie des schémas", le psychologue Jeffrey Young trace les schémas précoces d'inadaptation comportementale. Le psychologue explique dans sa théorie ce qu'est un schéma comportemental précoce inadapté:

"Les schémas pathologiques ${ }^{8}$ sont des structures mentales sélectionnées par un environnement (le passé) et devenues inadaptées à un autre environnement (le présent). Ils peuvent avoir présenté une valeur de survie dans l'histoire de l'individu ou bien celle de l'espèce dont ils représentent un vestige, qui a survécu à son utilité pratique". ( Joung, Thérapie des schémas, nouvelle édition,édition de l'homme, 2017,P. 14)

Joung explique que ces schémas sont ancrés dans les réseaux neuronaux de l'individu depuis l'enfance. Ce sont de fausses croyances enregistrés dans l'inconscient de la personne qui guident son comportement. Ces émotions emmagasinées servent à gérer son comportement actuel. Et si ces croyances sont fausses, elles empoisonnent le développement comportemental de l'individu. En des termes plus simples, les schémas pathologiques sont des structures mentales développés dans l'enfance comme un compromis pour pouvoir survivre dans un environnement malsain ou inadapté aux besoins fondamentaux de l'enfant . Par exemple, si un individu développe un schéma pathologique d'échec pendant l'enfance, cela traduit qu'il a souffert d'un environnement trop sévère de critique et de dévalorisation. Il développerait un complexe d'infériorité. Ces informations erronées sur soi-même fonctionnent dans l'inconscient comme des barrières au développement comportemental sain:

\footnotetext{
${ }^{8}$ Les schémas décrits par Young sont:: le schéma Abandon, le schéma Méfiance, Carence affective, Exclusion, Dépendance, Vulnérabilité, Imperfection, Échec, Assujettissement, Exigences élevées, Tout nous est dû.
} 
"Ils (schémas pathologiques) sont constitués de souvenirs, d'émotions, de pensées et de sensations corporelles. Ils concernent la personne et ses relations avec les autres. Ils se sont développés au cours de l'enfance et de l'adolescence. Ils se sont enrichis et complexifiés tout au long de la vie". (Young, thérapie des schémas, p. 15)

Ces schémas sont répétitifs, ce qui mène l'individu à répéter les mêmes erreurs comportementales,comme s'il était pris dans un engrenage de cercle vicieux:

"Les schémas précoces inadaptés se traduisent dans des comportements auto-défaitistes , qui (...) se répètent tout au long de la vie. " ( Young, thérapie des schémas, p. 16).

Vu l'importance du caractère répétitif de ces schémas pathologiques, Young souligne que " la plupart des patients porteurs de troubles de personnalité reproduisent, de façon autodéfaitiste, les modes de vie négatifs de leur enfance. Ils s'engagent en permanence dans des pensées, des émotions, des comportements et des types de relation qui maintiennent leurs schémas. De cette manière, ils poursuivent involontairement dans leur vie adulte la répétition des conditions de leur enfance qui ont été pour eux les plus néfastes. " (Young, Thérapie des schémas, p. 55)

De ce qui précède, nous pouvons déduire que ces déviations comportementales ne seront jamais complètement modifiés car elles sont inscrites dans le cerveau émotionnel, mais il y a un espoir que les comportements agressifs pourraient redevenir acceptables pour la société s'ils sont guidés à travers des modifications cognitives émotionnelles et comportementales.

Causes et origines des schémas pathologiques:

Schéma "Tout nous est dû" à fond impulsif:

Nous avons été surprise de la précision descriptive des failles comportementales présentées par la romancière et le psychologue. La superposition des cas présentés chez l'un et l'autre est vraiment surprenante de précision. Joung décrit 
le cas d'un malade soigné chez lui à la clinique. Voyons la similitude entre les cas:

"Maurice est imperméable aux sentiments des autres, comme la plupart des personnes qui estiment que tout leur est dî, tels des enfants gâtés. Leur égocentrisme est si profondément ancré qu'ils ne sont pas conscients de son effet sur leur entourage. (...) Il n'était pas conscient des moments où son comportement outrepassait les limites de la décence." ( Joung, Je réinvente ma vie, p. 343).

Le psychologue américain Jeffrey Joung, après avoir défini les symptômes des schémas pathologiques dans:" Thérapie des schémas, il a poursuivi ses études comportementales et les a publiées dans" Je réinvente ma vie ". Il y a précisé l'existence de onze schémas d'inadaptation sociale. Dans ce dernier ouvrage, il a précisé les symptômes et les origines de ces schémas. Nous avons découvert que les traits de burnerie détectés par la romancière correspondent au schéma intitulé "Tout nous est dû". Young n'a pas donné une définition fixe pour ses schémas, mais il a laissé le lecteur découvrir seul ses points de faiblesse à travers un style direct franc adressé d'emblée au lecteur de son ouvrage. Young à précisé 3 raisons essentielles comme origine du schéma : " Tout nous est dû".

1) Milieu éducatif permissif :

Comme son titre l'indique, la personne qui développe un tel schéma manque d'un cadre disciplinaire nécessaire à son mûrissement émotionnel au sein de sa famille. JOUNG précise:

"Par discipline, nous entendons l'instauration de règles raisonnables et des conséquences à leur manquement". (Joung, je réinvente ma vie, p. 331.

Le psychologue met en garde la société contre l'ambiance familiale relâchée. L'ambiance familiale trop permissive engendre chez la progéniture le sentiment de la supériorité. Elle développe un schéma comportemental égoïste :"Moi et 
mes besoins doivent passer avant les autres". Ce manque de respect pour les besoins des autres engendre un manque de maîtrise émotionnelle chez les enfants. Ils se croient les plus importants. Lorsque les parents gâtent leurs enfants (...) ces derniers viennent à croire que tout leur est d $\hat{u}^{\prime \prime}$ (je réinvente ma vie, p. 332)

2) Manque de mûrissement émotionnel chez les parents: Joung nous enseigne que le relâchement familial, et la permission de manquer à la discipline peut se transmettre également de père en fils: "Des adultes qui ne savent pas se maîtriser ne parviennent pas davantage à maîtriser leurs enfants. C'est en observant la discipline dont nos parents font preuve que nous apprenons la maîtrise de soi. Nous sommes tels qu'on nous a faits. Si nos parents nous imposent des limites claires, constantes et raisonnables, nous apprenons à nous les imposer à nous-mêmes. "( Young, je réinvente ma vie, p. 331)

Ainsi, la psychologie positive et la littérature mettent-elles en garde la société contre les ambiances familiales trop relâchées.

\section{3) contre-attaque :}

Joung explique que développer le schéma" tout nous est dû" peut également être une sorte de compensation à une lacune psychique. Comme le système nerveux cherche l'équilibre pour survivre, et s'il rencontre un manque crucial de ses besoins fondamentaux, il développe ce genre de comportement pour contre attaquer cette lacune.

" Malheureusement, l'éducation que nous avons reçue nous porte souvent à ne pas tenir compte de notre nature et à faire ce que l'on attend de nous. On nous oblige à la dureté quand nous sommes sensibles; on nous pousse vers des études de médecine quand nous sommes attirés par les activités d'extérieur; on nous inculque un comportement conventionnel quand tout nous incite au non conformisme ; on nous impose des tâches de routine quand le changement est ce qui nous stimule. " (Young, je réinvente ma vie, p. 354)

Des milliers d'exemples à citer de parents qui, avec les meilleurs intentions du monde, nous poussent à renier 
notre vraie nature, ce qui entrave l'épanouissement personnel et oblige l'inconscient à développer une contre attaque pour pouvoir survivre.

Nous supposons que chaque personnage dans le roman de Giordano ait souffert d'un drame familial qui a engendré ce genre de comportement. Maximilien, par exemple, à été élevé dans une ambiance de Rigidité paternelle, de froideur et d'abandon maternels. Il a souffert d'une carence affective majeure:

" Chez les Vogue, la gentillesse n'avait pas vraiment droit de cité. L'image de son père s'imposa un instant. Cet homme avait toujours encouragé la force et la fermeté. Ils étaient de ceux qui pensaient plus efficace la dureté que les encouragements. On ne faisait pas des hommes avec de la gentillesse. Un bon système de pression et ce qu'il faut de punitions, voilà ce qui donnait des résultats. Combien de fois s'était-il senti négativement irradié par l'état d'esprit de son père ? Jusqu'à quel point avait-il été contaminé ? "(corpus p. 86)

Maximilien Vogue a donc été élevé au biberon de la force. Aucun lien de tendresse ni de compréhension n'avait été tissé entre lui et son père. On lui a inculqué que la tendresse est une faiblesse :

"Il faut réaliser que j'ai été élevé au biberon de l'intransigeance et des rapports de force. Dans l'éducation que j'ai reçue, on m'a inculqué l'idée que développer de l'affectivité au travail peut être dangereux, ou perçu comme un aveu de faiblesse". (Corpus, p. 111.)

Pour développer une telle carapace de dureté émotionnelle, Maximilien à dû subir tout au long de sa vie l'impact négatif de son père :"Un homme ne pleure pas, un homme ne pleure pas... (....) Un homme se devait d'être fort. En toutes circonstances, un homme n'est pas là pour ressentir. Il est là pour agir". (corpus, p. 189).

Il se pose même la question si ce genre d'éducation pauvre en communication entre lui et son père n'était la principale raison de développer le schéma de la force, de la domination et de la burnerie ? : 
"Était-ce pour se protéger qu'il avait consciencieusement évité de laisser la place à ce type de sentiments dans sa vie ?" ( corpus, p. 190)

Malheureusement, le milieu familial de Maximilien était un milieu glacial. Ses besoins naturels et fondamentaux de communication, de tendresse n'étaient jamais assouvis. Souffrant d'un manque d'attention maternelle, Maximilien se remémore n'avoir jamais été dorloter par sa mère : "Mais ces neuf mois de gestation (période de grossesse) n'étaient ils pas les seuls moments vraiment maternels qu'elle avait eus à lui offrir ?"(corpus P. 161)

Lorsque son père lui rappelle qu'il doit téléphoner à sa mère pour prendre de ses nouvelles, Maximilien se souvient amèrement qu'elle ne lui avait jamais consacré du temps pour lui personnellement : "Pense à l'appeler un de ces jours. Elle me rabat les oreilles avec ça à longueur de temps. Quand elle n'est pas en train de faire un tournoi de bridge ou une séance de manucure, songea amèrement Max." (corpus, p. 204)

Cette carence affective grave engendre un sentiment de vide. Une lacune interne renvoie à l'image d'un enfant négligé:

"La carence affective est ce que ressent un enfant négligé". (Young, ⿺辶 réinvente ma vie, $P$. 126)

Cette carence affective le pousse par conséquent à compenser ce vide par un comportement exigeant. La lacune s'exprime par un contre attaque ou une compensation ce qui alimente l'égoïsme et le narcissisme. Devenir exigeant vis à vis de la société le calme intérieurement et comble son vide. "dans leur enfance, les besoins affectifs profonds de ces personnes n'étant pas comblés, les narcissiques ont appris à compenser leur carence en se montrant exigeantes à l'excès pour des besoins moins fondamentaux." (Young, Je réinvente ma vie, p.133)

De ce qui précède, nous déduisons que Maximilien a dû développer deux schémas d'inadaptation comportementale : la carence affective et Tout nous est dû. Il a compensé sa 
carence affective par le pouvoir, l'hégémonie et a calmé son vide par la célébrité et l'ambition :

"Vous compensez parfois la carence affective par l'ambition sociale. Vous tentez de combler votre vide affectif par le pouvoir, la célébrité, le succès, l'argent en substituant le prestige aux relations affectives enrichissantes". (Joung, je réinvente ma vie, P. 310)

De ce qui précède, nous constatons que les parents sont,parfois, responsables de la déviation comportementale de leurs progénitures. Non seulement, ils les affectent émotionnellement (la carence affective), mais les poussent également à développer un schéma pathologique pour pouvoir survivre (Tout nous est dû) :

"Certaines personnes présentant le schéma" carence affective" contre attaquent ; (...) elles se comportent comme si tout leur était dîn". P. 133.

Le personnage de Bruno a peut-être souffert d'un climat familial où les exigences de la réussite et de l'ordre étaient des exigences élevées. Il lui fallait atteindre des niveaux de perfection élevés au détriment du développement du bonheur. La recherche de la joie ou l'accomplissement raisonnable de soi étaient freinés par la rigidité des exigences. Lorsque les parents exigent des critères de réussite élevés au détriment des besoins fondamentaux de l'enfant, celui-ci risque de développer un schéma de perfection. Un cercle vicieux d'ambition ne cesse de ronger la personne intérieurement. Un démon interne qui ne se satisfait de rien. Joung explique que le schéma d'exigence élevée cherche le perfectionnisme:

"Vous foncez, foncez sans cesse, vous voulez toujours aller plus loin. Vous luttez pour être le meilleur en tout, que ce soit dans vos études, dans votre travail, dans les sports, dans vos loisirs, (...). " p. 305

Tout cela peut expliquer la dureté de Bruno envers ses collaboratrices qui n'avaient ni le souci de perfectionnisme, ni l'énergie, ni la disponibilité comme lui. Pour remédier à ses démons internes, Bruno contre attaque, lui aussi, par le schéma TOUT NOUS EST DÛ. 
Quant à Émilie, elle a été élevée dans une tradition bourgeoise rigide. Elle a vécu le mode de vouloir plaire aux parents pour se sécuriser contre les critiques. Elle avait, peut-être, craint le rejet ou l'abandon. Elle a développé un schéma d'ASSUJETISSEMENT pour prévenir les représailles : "Votre assujettissement provient de la certitude que vous devez plaire à vos parents, vos frères, vos sours, vos amis, vos professeurs, extra..." p. 273.

Pour prendre sa revanche de ses parents, elle projette son assujettissement sur son fils; elle contre-attaque par l'agressivité inconsciente envers son fils. Elle veut le dominer en lui imposant des études prestigieuses correspondant à l'image narcissique de sa caste élevée :

"De façon générale, les personnes assujettis sont plus à l'aise dans un rôle passif, mais certains d'entre elles composent avec leur schéma :" Assujetissement" en contre attaquant. Elles inversent les rôles et deviennent agressives et dominatrices. Elles surcompensent leur sentiment d'assujetissement par la révolte".p.278

Sa colère réprimée s'exprime indirectement de façon passive agressive. En projettant son schéma sur son fils, elle a voulu reprendre la même méthode d'éducation. Celui-ci a refusé catégoriquement d'être pris dans le même engrenage. Il a ainsi lutté pour l'expression de son identité et de sa liberté. JOUNG explique que: " l'assujettissement vous prive de votre liberté car tous vos choix sont dictés par les réactions d'autrui. (...) Vous ne vous dites pas :" voici ce que je veux et ce que je ressens", mais bien voici "ce que tu veux pour être content de moi". P. 272.

Alors,la réaction de son fils traduit son refus pour le chemin qu'elle lui trace: "c'est fini maman. Je reprends ma vie en main. Je ne te laisserai pas la foutre en l'air pour satisfaire ton petit ego de bourgeoise des familles." (corpus, p. 66)

Nous devons saluer la force de caractère de cet adolescent, qui, grâce à cette réaction agressive a réussi à préserver son identité d'un démantèlement probable. 
Selon les schémas décrits par le psychologue américain, Nous supposons que Nathalie avait développé pendant l'enfance le schéma "EXCLUSION". Elle a dû peut-être développer ce genre de comportement pour surcompenser une autre lacune psychique, une autre imperfection et un manque interne d'être accepté par l'entourage. YOUNG analyse ce cas ainsi: "Si vous affichez des prétentions excessives afin de compenser une Carence affective, (...) vos parents, froids et peu tendres, vous ont privé d'affection. Vous avez contre-attaqué en vous arrogeant tous les droits. (...) Devenu adulte, vous faites en sorte d'obtenir tout ce que vous voulez". ( Joung, Je réinvente ma vie, p. 333).

De peur de subir les mêmes privations en société, cette personne développe un narcissisme exigeant. Elle ne se satisfait intérieurement qu'après avoir semé la désaffection interne autour d'elle en société. Comme si elle projette son anxiété et sa peur sur l'entourage pour calmer son mal:

"une personne qui se sent indigne ou socialement indésirable peut compenser en se croyant unique. Si vous vous dites intérieurement: "je suis inférieur", il se peut que vous compensiez ce sentiment en vous persuadant que :"Non, je suis spécial, je suis meilleur que les autres." (Joung, je réinvente ma vie, p. 333).

Maintenant nous avons pu comprendre que ces personnages qui ont dû développé des stratégies dysfonctionnels d'adaptation à un milieu malsain voulaient se protéger d'une douleur secrète. Le développement de ces stratégies nocives n'a fait qu'aggraver la situation ; on est pris dans un cercle vicieux de répétition.

"Nous sommes condamnés à répéter les mêmes erreurs, à revivre ce qui nous a été légué par nos parents et nos grands parents si nous ne prenons pas la décision de faire le nécessaire (le processus de changement) pour remédier à la situation." (Joung, je réinvente ma vie, p. 352.) 
De ce qui précède, nous constatons que la romancière voulait aider ses personnages pour s'extraire à l'engrenage, rétablir l'équilibre interne d'un enfant saint:

" Le but final de l'approche est de parvenir à éprouver de la compassion pour l'enfant qu'il a été, ne plus l'accuser de tous les maux, ne plus le mépriser... Lui pardonner, se pardonner." ( Joung, soigner les schémas de pensée, p. 71.

Un adulte sain doit pardonner à ses parents, doit accepter que les lacunes d'éducation du passé ne doivent pas entraver son épanouissement futur. Il doit cesser d'avoir peur pour ne plus faire fonctionner ses schémas de compensations. Un adulte sain doit rejoindre intérieurement un enfant blessé, le comprendre et le calmer pour pouvoir s'épanouir émotionnellement .

\section{Conclusion :}

Notre recherche avait tenté de mettre en lumière un phénomène nouvellement parue dans les sociétés modernes. On ne savait pas pour quelle raison les gens sont devenus très agressifs les uns envers les autres.

Loin des maladies psychiques connus, la psychologie positive avait pour but de prévenir de succomber à la maladie ce qui a poussé les entreprises à prendre soin de leurs employés dans le but d'augmenter leurs rendements et leurs capacités productives.

Ce genre de prévention a poussé les psychologues à chercher les raisons profondes qui provoquent ce genre de déviation comportementale.

Diplômée d'étude psychologique, notre romancière a eu l'audace d'introduire dans la littérature ses méthodes de coach ou de créativité personnelle. Elle a réussi à présenter cinq cas différents dans leur milieu de travail et leur fonctionnement. Chacun avait ses propres raisons de 
succomber à la burnerie. Cependant, ils manifestaient le développement d'un même schéma pathologique. Malgré leurs bonnes intentions, Ils ont provoqué la souffrance autour d'eux sans comprendre qu'ils sont eux même les bourreaux et les victimes à la fois. Victime d'un milieu éducatif malsain, ils ont dû développer des schémas d'adaptation qui sont devenus par la suite des schémas d'inadaptation sociale. Lorsque la raison de la déviation à disparu, le cerveau émotionnel était saturé de mauvais traitement. Ce cerveau n'a pas pu effectuer les changements comportementaux nécessaires aux changements de leurs situations. Chaque cerveau a gardé ses moyens de défense de peur d'être attaqué de nouveau.

Nous avons eu la chance de découvrir la théorie du développement comportemental de Jeffrey Joung publié et expliqué dans son chef d'œuvre "Je réinvente ma vie". Le style simple de la vulgarisation scientifique a permis d'aborder les schémas pathologiques et les comprendre. Cela a parfaitement facilité notre tâche d'appliquer la théorie sur les cas présentés par la romancière. Celle-ci nous a permis de tirer au clair une des maladies sociales les plus répandues. Elle a expliqué le sens de la burnerie, comment en remédier à travers des exercices qui activent les cinq sens. Elle a mis en garde la société contre les raisons d'apparition de ce comportement nuisible. Elle a montré à travers son programme de coach comment doiton éveiller la conscience, la secouer pour voir clair le mal semé autour de la personne burnée. Elle a suivi principalement deux étapes. La première dite méthode cognitive pour que les ego trop enflés s'adaptent à l'humilité. Cette étape consistait à guider son groupe à se décentrer. La seconde consistait à savoir donner de soi, de son temps pour goûter au partage du bonheur. Elle les a initiés à se déconnecter du virtuel pour rejoindre la 
sensibilité humaine à travers les auvres de charité prodiguées aux orphelins.

L'analyse des schémas pathologiques des 5 personnages nous est parue d'une grande utilité pour fouiller les raisons de la déviation. Nous avons découvert, en fin de compte, que le milieu éducatif malsain est responsable de toute déviation comportementale.

Espérons avoir pu présenter une infime contribution à la compréhension de ce phénomène nocif. Espérons avoir pu prévenir la propagation des schémas pathologiques grâce à la compréhension de la problématique. 


\section{Bibliographie}

Le corpus:Le jour où les lions mangeront de la salade verte, Paris, Eyrolles, 2017.

Euvres de Raphaëlle Giordano: Les romans :

Ta deuxième vie commence quand tu comprends que tu n'en as qu'une, Paris, éditions Eyrolles, 2015.

Cupidon a des ailes en carton, Paris, Eyrolles, 2019.

Ouvrages de développement personnel:

Les secrets du docteur zen, 2010,

Faire face au changement, 2010,

Gestion du stress, 2010,

Mon carnet de coaching, 2012 ,

Jai décidé d'être zen, 2014,

L'affirmation de soi, 2010,

Les secrets du docteur coolzen,

Euvres de développement personnel ( basées sur la psychologie positive):

Brizendine (Louann DR.), Les secrets du cerveau féminin, Paris, Bernard Grasset, traduit de l'américain par Marie-France Girod.

Brouard (Stephanie) et Daverio (Fabrice), Les outils de développement personnel pour manager, Paris, Eyrolles, 2010.

Cyrulnick (Boris), et Alii, Votre cerveau n'a pas fini de vous étonner, livre de poche, Albin Michel. Paris,

Hirigoyen (Marie France), le harcèlement moral et la violence perverse au quotidien, Paris, Étude (poche), 2018.

Ogden (Jane), psychologie de la santé, De Boeck, Belgique, 2014, traduction d'olivier Desrichard et alii.

Oullier (Olivier) et Sauneron (Sara), Nouvelles approches de la prévention en santé publique, l'apport des sciences comportementales, cognitives et des neurosciences,édition AWS, Paris, 2010.

Rusineck (Stéphane), Soigner les schémas de pensée, Dunod, collection Psychothérapie, Paris, 2006.

Schnider (Armin), Neurologie du comportement, édition Masson, 2008. Traduit de l'allemand par Fabienne Perren. 
Young (Jeffrey), et Alii, la thérapie des schémas, approche cognitive des troubles de la personnalité, De boeck supérieur, the Guilford Press, 2017. Traduction française de Bernard Pascal.

Young, Klosko, Je réinvente ma vie, éditions de l'homme, Canada, 2013. Traduction de Marie perron.

ARTICLE :

Kauffman (Micheal), Les 7 P. De la violence masculine, Canada, Toronto, 1999. PP 1-8.

Thèses consultées :

Asdourian (Bruno), communication relation et médias sociaux, université Aix-Marseille II, 2010.

Bertin(Eric), Pour une sémiotique du champs stratégique de la communication, doctorat, université de Bourgognes, 2010.

Duguay (Benoît), l'image de soi et la consommation:la valeur compensatoire des produits, université du Québec, 2000.

Regourd-Laizeau (Martine), psychologie positive et EMDR: questions et perspectives, université de Lorraine, 2013.

Romma (Natacha), communication de changement comportemental sur le Web : comparer l'efficacité de persuasion et de l'engagement pour promouvoir l'éco-citoyenneté. Sciences de l'Homme, et Société, université du Sud Toulon Var, 2010.

Roussin (J.), psychothérapie selon l'approche des schémas de Young et analyse critique d'un processus thérapeutique, thèse de doctorat, université du Québec, 2011.

Salah-Eddine (Laila), le harcèlement moral au quotidien:analyse sociologique, thèse de doctorat, université Paris-Descartes, 2008.

Trépanier (Dominique), analyse d'une thérapie cognitive comportementale dans le traitement de la dépression, université du Québec à Trois-Rivières, 2010.

Dictionnaires consultés :

Le petit Robert

Le petit Larousse illustré 2017, Larousse, Paris, 2017.

Site Internet : www.raphaëllegiordano.com 